Service social

\title{
Améliorer la collaboration entre les milieux scolaire et de réadaptation : besoins des intervenants
}

France Beauregard et Jean-Claude Kalubi

Volume 57, numéro 2, 2011

URI : https://id.erudit.org/iderudit/1006301ar

DOI : https://doi.org/10.7202/1006301ar

Aller au sommaire du numéro

Éditeur(s)

École de service social de l’Université Laval

ISSN

1708-1734 (numérique)

Découvrir la revue

Citer cet article

Beauregard, F. \& Kalubi, J.-C. (2011). Améliorer la collaboration entre les milieux scolaire et de réadaptation : besoins des intervenants. Service social, 57(2), 158-172. https://doi.org/10.7202/1006301ar
Résumé de l'article

Différents auteurs ont écrit sur la nécessité pour les milieux de la santé, scolaire et familiaux de collaborer ensemble pour la réussite éducative de l'enfant présentant une déficience, un problème de santé ou une difficulté scolaire. Ces auteurs notent que les intervenants des milieux de la santé et scolaire ont des expertises différentes. Or, certains intervenants stigmatisent ces différences comme sources des difficultés à collaborer. L'étude présentée dans cet article porte sur les besoins identifiés par des intervenants des commissions scolaires de l'Estrie et ceux du Centre de réadaptation de l'Estrie relativement à leur collaboration. L'analyse des propos des participants a permis non seulement de répertorier leurs besoins, mais également de proposer des pistes de solution. 


\title{
Améliorer la collaboration entre les milieux scolaire et de réadaptation : besoins des intervenants
}

France Beauregard

Jean-Claude Kalubi

\section{RÉSUMÉ}

Différents auteurs ont écrit sur la nécessité pour les milieux de la santé, scolaire et familiaux de collaborer ensemble pour la réussite éducative de l'enfant présentant une déficience, un problème de santé ou une difficulté scolaire. Ces auteurs notent que les intervenants des milieux de la santé et scolaire ont des expertises différentes. Or, certains intervenants stigmatisent ces différences comme sources des difficultés à collaborer. L'étude présentée dans cet article porte sur les besoins identifiés par des intervenants des commissions scolaires de l'Estrie et ceux du Centre de réadaptation de l'Estrie relativement à leur collaboration. L'analyse des propos des participants a permis non seulement de répertorier leurs besoins, mais également de proposer des pistes de solution.

Mots-clés : Collaboration, besoin, réadaptation, scolaire, participation sociale.

\begin{abstract}
Numerous authors wrote about the need for the fields of health, school and family to work in collaboration for the educational success of children with disabilities, health problem or those having difficulties in school. They say that the professionals from the health agencies and the schools have different expertise. However, it seems some professionals stigmatize these differences as the main problem of working together. The study presented in this article addresses the needs the professionals of school boards of Estrie and the Centre de réadaptation de l'Estrie to meet in order to improve their collaboration. The analysis of comments made by participants has not only conducted to identify their needs, but also solutions.
\end{abstract}

Keywords: Collaboration, need, rehabilitation, education, social participation.

Plusieurs recherches ont porté sur la collaboration entre les familles d'enfants présentant des incapacités et l'école, ou encore entre ces familles et le milieu de la santé et de la réadaptation (Beckman, 2003; Beveridge, 2005; Blue-Banning, Summers, Frankland, Nelson et Beegle, 2004; Ulrich et Bauer, 2003). Ces recherches ont permis dans un premier temps de tracer un portrait de la situation et d'identifier des facteurs facilitant ou compromettant la collaboration famille-école et famille-réadaptation. Cependant, peu d'études concernent la collaboration milieu scolaire-milieu de la réadaptation. Pourtant, cette dernière est considérée par les parents comme un facteur facilitant qui allège leur tâche, puisqu'ils n'ont plus besoin de faire le lien entre les intervenants des deux milieux, ceux-ci communiquant directement entre eux (Beauregard, 2006; Bouchard et Kalubi, 2003). Notre étude porte donc sur les besoins à combler pour améliorer la collaboration entre les intervenants de ces deux milieux. Nous présenterons d'abord le contexte de recherche et le cadre théorique. Ensuite, nous décrirons la méthodologie et les résultats. Enfin, il y aura discussion des résultats et présentation de la conclusion. 


\section{CONTEXTE DE RECHERCHE}

Le Centre de réadaptation de l'Estrie (CRE) a mis en place un projet pilote, nommé Apollo (CRE, 2006), portant sur l'organisation des services en réadaptation pédiatrique. Ce projet a pris naissance à la suite de consultations auprès des usagers des services du CRE et de leur entourage, ainsi que du personnel concerné par le programme Enfants et adolescents. Celui-ci offre des services (ergothérapie, orthophonie, physiothérapie, aide psychosociale à la personne et à ses proches) s'adressant aux jeunes âgés de 0 à 18 ans qui ont des troubles de l'acquisition des tâches motrices et de l'élaboration des séquences de gestes, des encéphalopathies (incapacités motrices innées ou acquises, maladies neuromusculaires, myélopathies), des troubles spécifiques du langage et de la parole ou encore, des retards de développement global.

Les consultations auprès de cette clientèle ont montré que les services tels qu'organisés rendaient l'accessibilité difficile. Par conséquent, la liste d'attente pour l'obtention de services s'allongeait alors que les parents attendaient notamment du CRE une réponse rapide à leurs questions. Outre le fait d'accroître l'accès aux services par le projet Apollo, le CRE ciblait l'augmentation de la participation sociale de la clientèle en améliorant la collaboration avec le communautaire. Des mesures ont été mises en place pour atteindre ces objectifs. Ainsi, l'accueil de nouveaux clients et l'accessibilité à des services ont été facilités en offrant notamment des services de réadaptation de groupe plutôt qu'en individuel. Toutefois, les intervenants ont éprouvé des difficultés à établir une collaboration avec différents organismes, tels que les municipalités, les transports, les milieux de garde, les milieux scolaires, etc.

\section{Études sur la collaboration famille-école-réadaptation}

Différents auteurs ont écrit sur la nécessité pour les milieux de la santé, scolaire et familial de collaborer pour le bien-être de l'enfant présentant une déficience, un problème de santé ou une difficulté scolaire (Bouchard et Kalubi, 2003; Eppler et Weir, 2009; Turnbull, Turnbull, Erwin et Soodak, 2006). Outre le milieu familial, le milieu scolaire est celui où les enfants et les adolescents passent la plus grande partie de leur vie. Lorsqu'il s'agit d'enfants présentant une déficience physique, celui de la réadaptation s'ajoute à ces deux milieux. À cet égard, il est possible de trouver quelques études sur la collaboration entre les intervenants du milieu scolaire et ceux du milieu de la santé (Saaranen, Tossavainen et Turunen, 2005; Sezgin, 2009). Ces auteurs notent qu'avec les avancées en santé et l'ouverture de la société occidentale à l'égard des personnes ayant des besoins spéciaux, les intervenants des deux milieux sont de plus en plus appelés à collaborer. Cependant, ils constatent que certains besoins doivent être comblés pour que cette collaboration soit plus fructueuse, notamment en ce qui concerne la communication, les connaissances et les attitudes des intervenants.

Au Québec, deux études provenant du milieu de l'éducation ont procédé à une analyse des besoins des enseignants qui accueillaient dans leur classe un enfant recevant des services d'un centre de réadaptation. L'une s'est penchée sur les besoins de perfectionnement des enseignants travaillant auprès d'élèves déficients intellectuels profonds multihandicapés (Normand-Guérette et Frénette, 1993), alors que l'autre a analysé les besoins des enseignants 
reliés à l'intégration scolaire des enfants ayant subi un traumatisme cranio-cérébral (TCC) (Desaulniers, Beaupré, Lepage et Granger, 2005). Les auteurs de ces deux études observent que les enseignants disent avoir besoin de formation en lien avec la déficience ou le TCC, les différentes méthodes à mettre en place pour faciliter les apprentissages de l'élève, les conséquences de l'état de l'élève sur ses apprentissages, etc. De plus, ils souhaitent avoir de l'information sur l'élève lui-même, ses caractéristiques, ses forces, ses difficultés, son évolution. Enfin, ils expriment le besoin de recourir à un intervenant pivot qui ferait le lien entre les enseignants et le centre de réadaptation. Or, est-il nécessaire de le rappeler, aucune de ces études ne s'est penchée sur la collaboration entre les intervenants du milieu de la réadaptation et ceux du milieu scolaire.

\section{COLLABORATION ENTRE LES INTERVENANTS}

Si la collaboration entre tous les acteurs du milieu de l'éducation et du milieu de la réadaptation apparaît comme une nécessité, c'est surtout dans le but d'assurer le prolongement des effets de l'intervention dans les différents réseaux concernés (Epstein, 2001). La collaboration entre les intervenants s'actualise par les interactions qu'ils ont entre eux. Mais les structures organisationnelles propres à chaque milieu influencent cette collaboration. Et tout en travaillant dans un but commun, les individus, les groupes, les organisations ont des intérêts, des croyances et des valeurs diversifiés, voire parfois contradictoires (Arrendo, Shealy, Neale et LaPearl, 2004; Poursanidou, Garner et Watson, 2008; Sicotte, D'Amour et Moreault, 2002). Or la qualité de la collaboration entre intervenants est cruciale, pour la bonne raison qu'elle influe sur le fonctionnement des programmes d'intervention offerts et par le fait même sur le bien-être et le développement de chaque élève.

\section{Besoins à combler pour améliorer la collaboration}

L'existence d'une collaboration entre les différents intervenants repose-t-elle en grande partie sur l'évolution des besoins de la personne, l'évolution des besoins des intervenants et surtout l'engagement progressif de ces intervenants? Le besoin correspond en fait à la différence entre une situation vécue et une situation souhaitée par un individu ou un groupe d'individus (Pineault et Daveluy, 1995). La collaboration apparaît donc comme un processus social par lequel des acteurs sont appelés à partager la prise des décisions et le choix des mécanismes visant à combler leurs besoins. Les intervenants observeront une différence de degré de collaboration en fonction de leur niveau de sensibilité, leurs habitudes de vie ou de travail, leurs capacités, compétences et connaissances, ainsi que leur vision de ce qu'est la collaboration (Saaranen et al., 2005; St-Onge, Béguet et Fougeyrollas, 2002). La pratique de la collaboration repose essentiellement sur un engagement réel de la part des intervenants et sur l'importance accordée à la complémentarité des expertises de différents acteurs, des besoins explorés, des connaissances et des pratiques à exploiter, des règles organisationnelles facilitant le soutien mutuel, de la vision de leur autonomie dans la réalisation d'un programme (Dinnebeil, Hale et Rules, 1996). L'analyse des besoins auxquels apporter une réponse pour améliorer la collaboration permet de constater la nature de ces derniers et la place qu'ils occupent dans les interactions de collaboration entre les acteurs du milieu scolaire et ceux du milieu de la 
réadaptation, donnant ainsi la possibilité d'élaborer des pistes de solution pour favoriser une collaboration plus fructueuse.

En définitive, il est nécessaire d'aborder la collaboration comme un cheminement interactif fondé sur les efforts de chacun pour expliciter ses réalités, afin que chacun puisse en faire une appropriation en tenant compte de la reconnaissance de ses alliances, de ses buts, de ses méthodes, de ses responsabilités et de ses exigences de communication. II s'agit donc dans cette recherche de déterminer les besoins que les intervenants du milieu scolaire et du milieu de la réadaptation souhaitent voir combler pour que leur collaboration soit plus fructueuse, puis de proposer des mesures qui répondent à ces besoins.

\section{MÉTHODOLOGIE}

Notre problématique, notre cadre conceptuel ainsi que notre question spécifique de recherche nous orientent vers une recherche de type qualitatif et descriptif (Savoie-Zajc, 2000; Van der Maren, 1995). En effet, cette étude cible l'identification de besoins à combler chez des intervenants provenant de deux milieux pour améliorer leur collaboration. Pour des raisons de logistique (disponibilité, regroupement, temps...), deux outils ont été privilégiés pour la cueillette de données. Ainsi, les intervenants du CRE ont participé à un groupe de discussion, alors que les intervenants du milieu scolaire ont répondu à un questionnaire.

\section{Intervenants du Centre de réadaptation de l'Estrie}

La personne responsable du programme Enfants et adolescents du Centre de réadaptation de l'Estrie a fait part de la recherche à ses intervenants. Douze intervenantes, toutes rattachées au siège social du Centre, ont mentionné leur intérêt à participer à une telle étude. II a alors été facile de les regrouper pour une entrevue. Ces intervenantes provenaient de différents groupes professionnels : orthophonie, ergothérapie, physiothérapie, technique en éducation spécialisée, travail social. Elles avaient toutes plus de dix années d'expérience dans le milieu scolaire et travaillaient en collaboration avec différents intervenants des commissions scolaires francophones de l'Estrie. Des thèmes tels que les difficultés rencontrées lors des rencontres avec les intervenants du milieu scolaire, les pratiques gagnantes et les besoins qu'elles aimeraient voir combler ont été abordés lors du groupe de discussion. Ces thèmes étaient ceux que les intervenantes du CRE désiraient approfondir. La discussion de groupe a eu lieu au CRE et a duré trois heures. Elle a été enregistrée sur bande audio et transcrite par la suite.

\section{Intervenants du milieu scolaire}

Les intervenants du milieu scolaire étant, pour leur part, dispersés dans toute la région de l'Estrie, l'envoi d'un questionnaire anonyme a été favorisé. Une fois dûment rempli, les intervenants le retournaient à la chercheuse. Ce fonctionnement assurait au répondant la confidentialité de la démarche. Le questionnaire était divisé en deux sections. La première section formée de questions fermées permettait de tracer un portrait des participants (nombre d'années d'expérience, formation, etc.), alors que la deuxième section était constituée de questions ouvertes qui demandaient d'identifier leurs besoins à combler pour améliorer la collaboration avec les intervenants du CRE. Plus de 150 questionnaires ont été envoyés, 49 ont 
été retournés, ce qui donne un taux de réponse de $30 \%$, jugé satisfaisant (Creswell, 2002). Les participants du milieu scolaire provenaient des trois commissions scolaires francophones de la région de l'Estrie: Commission scolaire régionale de Sherbrooke (67,3\%), Commission scolaire des Sommets $(20,4 \%)$ et Commission scolaire des Hauts-Cantons (8,2\%). Les données sociodémographiques montrent que les répondants correspondent à ce que l'on retrouve dans la réalité scolaire : une majorité des participantes sont des femmes (85\%), l'âge moyen est de 40-49 ans et moins de $15 \%$ des participants ont moins de 5 ans d'expérience dans le milieu scolaire. Enfin, on retrouve presqu'autant d'enseignants $(n=12)$ que de directions d'école $(n=11)$ ou de techniciens en éducation spécialisée (TES, $n=11)$. Les autres intervenants sont des préposées $(n=6)$, orthopédagogues $(n=2)$, psychologues $(n=4)$ et autres $(n=3)$. Ils interviennent principalement au primaire $(59,2 \%)$ et au secondaire $(22,4 \%)$. Enfin, près du tiers des participants travaillent en classe ordinaire (30,6\%), environ un cinquième en classe spéciale (22,4\%), alors qu'un quart des participants travaillent à la fois en classe ordinaire et en classe spéciale $(24,5 \%)$ et que $12,2 \%$ des participants interviennent dans une école spéciale.

\section{Analyse des données}

La méthode d'analyse privilégiée pour cette étude est l'analyse de contenu. De nature qualitative, elle permet de décrire, de clarifier, de comprendre ou d'interpréter une réalité (Denzin et Lincoln, 2005). Différentes méthodes peuvent être utilisées pour cette analyse. L'analyse thématique continue proposée par Paillé et Mucchielli (2008) nous a semblé la plus adéquate pour cette étude puisqu'elle fait appel à une logique inductive. Elle permet de regrouper une série de thèmes correspondant à des définitions qui peuvent être facilement identifiées dans le contenu des discours des participants. Nous avons ainsi regroupé les données selon chaque milieu. Quatre catégories de besoins ont émergé des propos des intervenants. Deux catégories, connaissances et attitudes, sont communes aux deux groupes d'intervenants, alors que les troisième et quatrième catégories sont propres à chaque milieu : règles administratives pour les intervenants du CRE et soutien pour les intervenants scolaires. De chaque catégorie, nous avons pu, par la suite, dégager des sous-catégories différentes selon le milieu et cibler des interventions potentielles pour l'amélioration de la collaboration. Enfin, afin de mieux saisir l'occurrence des thèmes, nous avons compilé le nombre d'intervenants et le nombre de fois que ces derniers faisaient allusion à un thème.

\section{RÉSULTATS}

Nous présenterons d'abord les résultats concernant les intervenantes du CRE, puis ceux des intervenants du milieu scolaire.

\section{Intervenantes du CRE}

Trois catégories de besoins pour les intervenantes du CRE ont été identifiées. L'occurrence des thèmes a permis de classer ces catégories en ordre de priorité. Ainsi, lors de la discussion de groupe, chaque participante s'est exprimée à huit reprises sur le besoin de connaissances des intervenants du milieu scolaire, cinq fois sur les règles organisationnelles et à quatre reprises sur les attitudes des intervenants du milieu scolaire. 


\section{Connaissances}

Le besoin que mentionnent le plus souvent toutes les intervenantes du CRE est celui des connaissances. En effet, elles soulignent le manque de connaissances des intervenants du milieu scolaire, notamment des déficiences (physiques, sensorielles, capacités et incapacités de l'élève), des ressources, des outils technologiques et du matériel adapté. Elles se questionnent entre autres sur la formation que ceux-ci reçoivent à l'égard de ces élèves. Une autre cause, à leurs yeux, qui explique la méconnaissance des intervenants scolaires à l'égard de leur clientèle concerne l'absence de transfert d'information entre les intervenants scolaires. II peut s'agir de dirigeants (directions d'école, services éducatifs, conseillers pédagogiques, etc.) qui participent à des séances d'information et de formation et qui ne transmettent pas les savoirs acquis à leur personnel enseignant. Il peut s'agir de manque de transfert d'informations concernant un élève handicapé d'un enseignant à un autre enseignant, d'une direction d'école au personnel de soutien à l'enfant (ex. : technicien en éducation spécialisée) ou lors de l'arrivée d'un nouveau membre à l'école. Ou encore, de l'envoi d'un dépliant sur une déficience qui n'est pas diffusé auprès des intervenants concernés. Bref, elles observent que les intervenants scolaires échangent peu l'information qu'ils ont sur l'enfant. Conséquemment, pour elles, tout est à refaire chaque année.

Quand quelqu'un a les connaissances et que ce n'est pas partagé, je trouve cela triste. Ça fait que chaque personne réinvente la roue chaque année alors qu'il y a déjà des choses de faites. (Intervenante P)

Dans le même ordre d'idées, des intervenantes remarquent que dans certains cas l'accessibilité au dossier de l'élève handicapé n'est pas facile. Que ce soit à cause d'horaires de travail incompatibles (ex. : direction absente lorsque la technicienne en éducation spécialisée est présente), du transfert d'école ou de toute autre raison, à leurs yeux, c'est une perte d'information. Le manque ou l'absence de connaissances et de transfert d'informations compliquent, selon elles, la collaboration avec les intervenants du milieu scolaire. D'abord, les intervenantes du CRE observent que chaque année elles doivent redonner la même information sur l'élève handicapé. Pour elles, un temps précieux est perdu alors qu'il pourrait être utilisé autrement, notamment pour soutenir les intervenants du milieu scolaire. Elles constatent en outre que le transfert d'informations pourrait combler certaines lacunes quant à la formation des intervenants du milieu scolaire. Bref, pour elles, le premier besoin à combler pour améliorer la collaboration entre les deux milieux est l'augmentation des connaissances ou, du moins, un meilleur partage des informations.

\section{Règles organisationnelles}

Le deuxième besoin est une meilleure compréhension des règles administratives. En effet, elles constatent que les règles sont parfois différentes d'une commission scolaire à l'autre, d'une école à l'autre. Par exemple, les intervenantes du CRE remarquent qu'elles reçoivent souvent de l'information différente, voire contradictoire, d'un endroit à l'autre. Ou encore, elles obtiennent de l'information qui ne concorde pas avec ce qu'elles lisent dans les écrits officiels. Elles s'interrogent notamment sur les règlements administratifs invoqués par certains administrateurs pour expliquer l'absence de transfert d'information, notamment le refus de 
donner accès au dossier sous prétexte de confidentialité alors que les parents ont signé le formulaire donnant leur accord. II peut s'agir de l'absence de services à un élève relevant du territoire desservi par le CRE sous prétexte d'un manque d'argent.

On a un document du ministère de l'Éducation au niveau des services pour l'enfant handicapé avec des codes. Selon ce document, cette enfant-là obtient le code XX pour lequel le soutien est décrit comme continu. Elle n'a rien, zéro. [...] Le directeur pédagogique regarde avec la direction pour répondre aux besoins de l'enfant, mais sans donner d'argent. Pourtant cette enfant, elle doit en générer des sous, c'est sûr, elle a le code XX. (Intervenante O)

Les intervenantes du CRE ont alors l'impression que des choix sont faits selon le budget et non pas selon les besoins de l'élève handicapé. Ces réflexions les amènent à s'interroger entre autres sur la compréhension et l'interprétation que les intervenants du milieu scolaire ont de leurs propres règles administratives. De plus, elles constatent que pour un même budget, des directions d'école «font des miracles », alors que d'autres non. Pour les intervenantes, ces informations contradictoires rendent la collaboration difficile. Ces dernières souhaitent donc que les règles administratives soient harmonisées d'un endroit à un autre. Dans le cas contraire, elles ont besoin de comprendre la raison de ces divergences.

\section{Attitudes $^{\underline{1}}$}

Le troisième besoin décrit par les intervenantes du CRE touche les attitudes des intervenants du milieu scolaire. D'abord, elles remarquent que ces derniers vivent des difficultés que nous venons de décrire (transfert de connaissances, ressources différentes d'un endroit à l'autre...). Les intervenantes ajoutent que leurs conditions de travail ne sont pas faciles : ratio élèvesenseignante élevé, ratio élèves HDAA plus élevé, des cas trop lourds pour la classe ordinaire, roulement du personnel, etc. Cependant, ces situations étant similaires pour tous les intervenants du milieu scolaire, elles s'interrogent sur le fait que la collaboration est facile avec certains et difficile avec d'autres. Les intervenantes du CRE remarquent que les premiers communiquent rapidement avec elles et s'informent sur l'enfant, alors que les derniers les contactent seulement en situation d'urgence. Elles soulignent également que certains intervenants sont réticents à introduire de nouvelles interventions, soit par méconnaissance, soit parce que ces dernières vont à l'encontre de leurs croyances.

Pour les intervenantes du CRE, la difficulté à collaborer dans certains cas s'explique non seulement par le manque de connaissances et d'harmonisation des règles, mais également par la différence culturelle entre les deux milieux. En effet, pour elles, les deux milieux n'interprètent pas la situation de la même manière. Là où l'intervenant du milieu scolaire voit une difficulté et une injustice par rapport aux autres, pour elles, il s'agit des caractéristiques de l'élève handicapé et d'équité.

1. L'attitude est définie comme un état mental de préparation à l'action organisée à travers l'expérience, exerçant ainsi une influence directive sur le comportement (Allport, 1935, dans Pratkanis, Breckler et Greenwald, 1989). 
Eux, ils partent du programme du Ministère, après c'est l'élève : « Qu'est-ce que je fais pour que l'élève réussisse le programme? » Nous, ce n'est pas l'élève. C'est l'enfant en premier, le plan d'intervention après. On a au départ des visions différentes. (Intervenante $\mathrm{E}$ )

Des intervenantes du CRE ajoutent que cette différence se retrouve dans le sens donné aux mots tels qu'autonomie, persévérance, attente réaliste, confidentialité. Difficile dans de telles conditions de communiquer adéquatement. Dans le même ordre d'idées, elles souhaitent que certains intervenants scolaires aient des attitudes plus ouvertes à l'égard des parents. Par exemple, des parents les appellent souvent en renfort lors de rencontres telles que l'élaboration du plan d'intervention adapté, parce que ces derniers se sentent jugés, ont l'impression que leurs demandes sont considérées comme excessives, etc. Ils interpellent alors les intervenantes du CRE pour leur soutien, pour qu'elles les rassurent et enfin qu'elles les aident à défendre les droits de leur enfant. Pour celles-ci, un intervenant scolaire qui manifeste des attitudes d'ouverture facilite la collaboration entre les deux milieux, car la communication est alors positive.

\section{Intervenants du milieu scolaire}

Tout comme pour les intervenantes du CRE, les catégories sont présentées par ordre d'importance, déterminée à partir du nombre de participants qui s'est exprimé pour chaque catégorie. Ainsi, 39 intervenants ont rédigé des commentaires sur les besoins de connaissances, 34 intervenants se sont exprimés sur le besoin de soutien et enfin 17 intervenants ont commenté les attitudes des intervenantes du CRE.

\section{Connaissances}

À l'instar des intervenantes du CRE, le besoin sur lequel s'expriment le plus les intervenants du milieu scolaire est une augmentation de leurs connaissances à l'égard de la clientèle desservie par le CRE. Cela concerne autant le handicap, les conséquences de ce dernier sur les apprentissages, les moyens et les outils d'adaptation, que les connaissances au sujet de l'élève, soit son évolution, ses progressions et ses régressions, ses évaluations, ses différents diagnostics, etc. II faut noter que les besoins de connaissances varient selon la fonction professionnelle. Par exemple, le corps enseignant souhaite de l'information sur l'apprentissage de l'enfant handicapé et les outils pédagogiques qui l'aident alors que les techniciens en éducation spécialisée et les préposés veulent recevoir des formations par exemple sur l'insertion sociale du jeune, son alimentation, sa sécurité physique. Quelques intervenants mentionnent que les formations ne répondent pas toujours à leurs besoins d'informations. II peut s'agir par exemple d'une formation sur une déficience qui s'adresse à tous les enseignants, mais cible uniquement une population spécifique au primaire. Ou encore, la formation donnée n'est pas en lien avec les apprentissages scolaires ou les approches pédagogiques favorisées par l'école. Par ailleurs, plusieurs intervenants considèrent qu'une formation de quelques heures n'est pas suffisante. Ces derniers souhaitent avoir des contacts subséquents avec les intervenants du CRE. Ils s'assureraient ainsi que leur compréhension de la situation et leurs interventions sont adéquates. 
On a besoin d'un suivi de l'élève et [d'une] communication relative à ses besoins en relation avec le vécu scolaire et les interventions du CRE; maintenir une continuité de rencontres (minimum 4) annuelles avec tous les intervenants de l'élève; favoriser l'acquisition des connaissances nécessaires pour mieux guider l'élève à l'intérieur de toutes les compétences qu'il a à développer. (Direction d'école)

Plusieurs intervenants veulent non seulement acquérir ces connaissances, mais également savoir où et comment aller les chercher. De plus, près de $70 \%$ des intervenants du milieu scolaire mentionnent qu'une façon d'augmenter les connaissances est le transfert d'informations sur l'élève handicapé. Selon eux, toutes les personnes concernées par l'élève devraient connaître les informations le concernant. Ainsi, ils voudraient une présentation du portrait du jeune dès le début de l'année scolaire et non pas attendre qu'il y ait une difficulté pour parler de ce dernier. Notons que ce ne sont pas tous les intervenants scolaires qui souhaitent ce type de rencontre. En effet, certains enseignants préfèrent voir évoluer l'élève dans leur classe afin que leurs perceptions ne soient pas teintées d'idées préconçues.

Enfin, des intervenants, rarement invités, voudraient pouvoir participer aux rencontres où l'on discute de l'élève. II s'agit plus particulièrement de professionnels, d'enseignants spécialistes, de techniciens en éducation spécialisée et de préposées. Ainsi, leurs connaissances sur l'élève seraient mises à jour. Selon eux, ce serait l'occasion non seulement de préciser les tâches et les responsabilités de chacun, mais également de connaître et de reconnaître l'expertise de tous. Un psychologue note entre autres que cette participation lui permettrait par la suite d'offrir du soutien et des conseils selon des problématiques précises. Bref, mettre en place des moyens pour augmenter les connaissances faciliterait la collaboration non seulement entre les intervenants des deux milieux, mais entre les intervenants d'un même milieu.

\section{Soutien}

Le deuxième besoin que les intervenants évoquent le plus souvent est celui du soutien. II est de deux ordres. Le premier type de soutien concerne le matériel adapté pour l'enfant. II peut s'agir tant de manuels et d'outils pédagogiques que de plan de travail, de crayon avec embout, d'ordinateur. Le deuxième type de soutien vise les intervenants du CRE. Outre l'augmentation de services tels que orthophonie, ergothérapie, physiothérapie, les intervenants scolaires veulent pouvoir consulter facilement les intervenants du CRE pour différentes raisons, par exemple pour être conseillés lors de l'achat et de la mise en place de matériel ou pour obtenir une information sur une problématique précise. Si certains intervenants du milieu scolaire considèrent que les intervenants du CRE montrent une disponibilité à cet égard, d'autres sont plutôt d'avis qu'il est difficile de rejoindre des intervenants du CRE.

Par ailleurs, quelques intervenants ont exprimé le besoin de groupes d'échanges comme mesure de soutien. Ce serait, pour eux, l'occasion de discuter de différents problèmes qu'ils rencontrent, des solutions qu'ils ont trouvées ou non, de bons et moins bons coups. Ce serait également l'occasion de réfléchir sur les besoins des jeunes handicapés, leurs intérêts, leur motivation, leur bien-être. Aussi, certains souhaitent que ce groupe soit hétérogène, c'est-à-dire 
que les différents professionnels travaillant avec les élèves handicapés (enseignants, orthopédagogues, TES, préposés, psychologues, orthophoniste, etc.) soient ensemble.

Des mini-rencontres multi (profs concernés - TES - PEH - psychologue) à souhaiter au moins 3 fois/année début d'année - mi-année et fin d'année. Buts objectifs : voir le profil de l'élève, revoir le P.I., cibler les balises à respecter, les objectifs à poursuivre, voir les embûches pour s'assurer que le P.I. de l'élève est bien respecté comme outil de travail. Ainsi, cela permettrait que chacun sache ce que les autres font avec leurs élèves handicapés et de poser différents regards pour une même situation. (TES)

\section{Attitudes}

Plusieurs intervenants du milieu scolaire soulignent le besoin de collaboration et veulent être impliqués dans les différentes démarches concernant l'élève handicapé. Si certains se disent satisfaits de la collaboration, plus de la moitié ont des réserves à cet égard. Certes, il y a les obstacles usuels (disponibilité, horaire de travail, ressources), mais les intervenants du milieu scolaire font également allusion aux attitudes des intervenants du CRE. Ainsi, plusieurs notent qu'ils ne sont pas toujours consultés lors d'une prise de décision. Cette situation leur déplaît, particulièrement lorsque la décision concerne leur tâche. Ils ont alors l'impression qu'ils se font dire quoi faire et comment le faire plutôt que d'être dans une relation de collaboration. D'autres notent qu'ils ne sont pas au courant des recommandations, conséquemment, ils ne les appliquent pas adéquatement et leur travail est alors critiqué. Ils s'interrogent d'ailleurs sur le fait qu'ils ne sont pas consultés quant à l'information complémentaire qu'ils pourraient apporter.

Parallèlement, quelques intervenants soulignent qu'il faut tenir compte du contexte actuel, à savoir du roulement de personnel, du manque de disponibilité de certains professionnels, d'une clientèle plus lourde et d'un manque de temps pour s'approprier et appliquer les nouvelles connaissances. Une enseignante note également qu'il y a dans sa classe des élèves qui vivent des difficultés plus grandes que l'élève handicapé et qui, pourtant, ne reçoivent aucun service du CRE ou d'autres ressources. Enfin, certains commentaires émanant des intervenantes du CRE sont perçus comme un jugement porté sur leur travail pédagogique et leur relation avec la famille de l'élève handicapé.

Mes quelques interventions avec un intervenant du CRE ont toujours débuté sur la défensive, car l'intervenant, toujours en lien avec la famille, vient en quelque sorte valider les perceptions des parents et on se sent jugé. On tient peu compte des limites du milieu et nos limites sont souvent interprétées comme de la mauvaise volonté. (Enseignante)

Bref, pour ces intervenants, une façon d'améliorer la collaboration est de mieux comprendre leur réalité et de voir qu'ils font du mieux qu'ils peuvent compte tenu des circonstances.

\section{Discussion}

Des auteurs ont relevé l'importance de la collaboration entre les différents milieux pour la réussite éducative et sociale des enfants (Epstein, 2001; Turnbull et al., 2006). Ils ont identifié 
des conditions essentielles pour que cette collaboration soit fructueuse. Certaines sont d'ordre structurel (telles les règles administratives, la logistique de travail), tandis que d'autres sont d'ordre individuel (comme les croyances, les attitudes). La satisfaction des intervenants à l'égard de leur collaboration avec d'autres intervenants sera en lien avec le respect de ces conditions. Conséquemment, le non-respect de celles-ci conduira des individus à exprimer des besoins, car ces derniers, tels que les définissent Pineault et Daveluy (1995), correspondent à la différence entre ce qu'ils vivent et ce qu'ils souhaitent vivre.

Les résultats de notre étude confirment ces conclusions. Si les intervenants du milieu de la réadaptation et du milieu scolaire s'accordent pour dire qu'il existe une certaine collaboration entre eux, plusieurs décrivent des situations au cours desquelles cette dernière est plus difficile. À la lecture des résultats, on comprend que ces difficultés sont reliées en grande partie à des connaissances, à des pratiques professionnelles ainsi qu'à des règles organisationnelles (Dinnebeil et al., 1996). Ainsi, les intervenants se sont exprimés à plusieurs reprises sur le besoin de connaissances. Les connaissances visées variaient selon le groupe. Ainsi, les intervenants du milieu scolaire ont exprimé le besoin de mieux connaître les déficiences, les outils, les conséquences sur l'élève, alors que le besoin de connaissances exprimé par les intervenantes du CRE concernait les règles administratives. D'autres commentaires faisaient plutôt allusion aux attitudes de chacun - aux jugements portés à leur égard ou à l'endroit de l'élève ou de sa famille. De plus, des intervenants ont émis des commentaires sur l'organisation qui différait d'un milieu à l'autre, rendant ainsi la collaboration plus difficile. Ces divergences de pratiques professionnelles et de règles organisationnelles font dire aux intervenants qu'ils ont des cultures différentes. Or, des études ont montré que la vision et les habitudes de travail propres à chaque milieu influent sur le degré de collaboration (Arrendo et al., 2004; Saaranen et al., 2005; Sicotte et al., 2002; St-Onge et al., 2002).

Par ailleurs, l'analyse des résultats montre qu'à l'intérieur d'un même groupe certains besoins sont communs à tous les individus appartenant à ce groupe alors que d'autres sont particuliers à un petit nombre d'individus et même à un individu. Par exemple, les intervenants du milieu scolaire souhaitent obtenir de l'information sur l'élève. Cependant, certains désirent l'obtenir avant de connaître l'élève alors que d'autres préfèrent enseigner à l'élève d'abord quelques semaines puis avoir de l'information sur ce dernier, ce qui suppose plus d'une façon pour diffuser cette information. Les préoccupations des uns ne sont donc pas nécessairement celles des autres, et les solutions à mettre en place pour combler les besoins devraient en tenir compte. Autrement dit, il est fort probable qu'il y aura plus d'une façon de répondre à un besoin. De plus, il est possible de remarquer que les besoins sont interreliés, c'est-à-dire qu'un besoin peut découler d'un autre. Par exemple, le besoin de compréhension des règles administratives exprimé par les intervenants du CRE rejoint, en fait, un besoin de connaissance de ces règles, tout comme le besoin de soutien et de suivi exprimé par les intervenants scolaires dépend des règles organisationnelles des deux milieux. En déterminant les besoins des intervenants quant à leur collaboration, besoins reliés aux savoirs, savoir-faire et règles organisationnelles, il devient alors possible de proposer des mesures adéquates.

Bien sûr, il existe de la formation continue, mais celle-ci pourrait prendre différentes formes. Une formation, des journées d'étude, des colloques, organisés en concertation soit par le 
ministère de la Santé et des Services sociaux et le ministère de l'Éducation, du Loisir et du Sport, soit par le Centre de réadaptation et les commissions scolaires de l'Estrie, permettraient de réunir les intervenants des deux milieux. Outre l'acquisition de connaissances, ces évènements seraient l'occasion de réfléchir et d'échanger, notamment sur les habitudes de travail et les règles organisationnelles. Cela permettrait également de sensibiliser les uns et les autres aux réalités de chacun et aux attitudes qui facilitent la collaboration ou nuisent à celle-ci. Ce type d'évènement pourrait regrouper les étudiants de formation initiale en enseignement et en réadaptation pour qu'il y ait un premier contact.

D'autres mesures concerneraient plutôt le transfert d'information, que ce soit en facilitant l'accès au dossier de l'enfant pour le personnel scolaire, ou encore, en permettant à des intervenants qui travaillent auprès d'un enfant de se rencontrer régulièrement pour échanger sur ce dernier. Ces mesures demanderaient un ajustement au niveau des règles organisationnelles et habitudes de travail. Dans le même ordre d'idées, un intervenant pivot (par exemple une orthopédagogue ressource) qui ferait le lien entre le milieu scolaire et le milieu de réadaptation pourrait assurer le transfert de connaissances. Des études ont d'ailleurs montré que c'est une mesure réclamée par plusieurs enseignants (Normand-Guérette et Frénette., 1995; Desaulniers et al., 2005).

Enfin, un moyen qui pourrait être mis à profit bien davantage est l'utilisation des sites internet. En effet, chaque centre de réadaptation a un site internet. On pourrait retrouver par exemple sur ces sites de l'information sur les déficiences, leurs conséquences sur la vie de l'enfant et celle de sa famille, les différents corps professionnels et leur rôle, des propositions d'outils. Un forum pourrait être exploité au sein du site. Les intervenants scolaires pourraient y poser des questions et obtenir une réponse rapidement. De leur côté, les commissions scolaires pourraient mettre des informations pertinentes en lien avec les élèves handicapés sur leur propre site internet, telles que les règles qui les concernent et les services offerts, de même qu'un hyperlien permettant l'accès au site du centre de réadaptation. Bref, différentes mesures peuvent être mises en place pour répondre aux besoins des intervenants. Cependant, il sera important avant la mise en place d'une mesure ou d'un moyen, de tenir compte du contexte et de la réalité de chaque milieu. De plus, l'amélioration de la collaboration ne reposera pas sur les épaules d'un seul milieu, d'une seule mesure. C'est l'ensemble qui en assurera le succès.

\section{Conclusion}

Cette recherche a permis de déterminer quels besoins des intervenants du milieu de la réadaptation et du milieu scolaire devaient être comblés pour améliorer leur collaboration. Les résultats sont similaires à ceux obtenus par d'autres études québécoises. En ajoutant le volet des intervenants du milieu de la réadaptation, nous avons pu obtenir un portrait plus précis. En effet, nous avons vu que des besoins sont communs aux deux groupes d'intervenants et d'autres spécifiques à chaque groupe. Nous avons pu proposer ensuite des mesures pour favoriser la collaboration entre les deux milieux. Notons que depuis cette étude, quelques mesures ont été mises en place, notamment une journée d'étude concertée, et que le site internet du CRE a été modifié pour permettre une meilleure diffusion de l'information. Une étude 
de l'impact de ces mesures sur la collaboration entre les intervenants des deux milieux serait à faire.

Comme toute autre recherche, celle-ci comporte certaines limites. D'abord, on ne peut pas généraliser les résultats obtenus à l'ensemble des intervenants, que ce soit du CRE ou du milieu scolaire, parce que le nombre de participants n'est pas suffisant. Les avis exprimés par les participants au sujet de leur collaboration ne représentent pas nécessairement ceux de l'ensemble des intervenants du CRE et du milieu de scolaire. En effet, nous ne connaissons pas les motifs pour lesquels les autres intervenants n'ont pas participé à cette étude. Cependant, l'objectif de cette recherche n'était pas de généraliser les résultats à l'ensemble des intervenants, mais de mieux saisir une situation. Enfin, les méthodes de collecte de données différentes selon le groupe ne nous permettent pas de tirer des conclusions concernant certaines notions. En effet, en contexte de groupe de discussion, les idées d'une personne peuvent avoir une influence sur les propos des autres, ce qui n'est pas le cas lors d'un questionnaire rempli à la main, tel que cela a été fait avec les intervenants du milieu scolaire. Cela dit, les données recueillies nous ont permis de remplir les objectifs de recherche qui étaient de déterminer des besoins que les intervenants du milieu scolaire et du milieu de la réadaptation souhaitent voir combler pour améliorer leur collaboration, puis de proposer des mesures qui répondent à ces besoins.

France Beauregard, Ph.D. Professeure adjointe Faculté d'éducation DEPP,Université de Sherbrooke

Jean-Claude Kalubi, Ph.D. Professeur titulaire Faculté d'éducation Université de Sherbrooke 


\section{RÉFÉRENCES BIBLIOGRAPHIQUES}

Arrendo, P., C. Shealy, M. Neale et L. W. LaPearl (2004). «Consultation and interprofessional collaboration: Modeling for the future », Journal of Clinical Psychology, 60(7), p. 787-800.

Beauregard, F. (2006). "Représentations sociales des parents et des enseignants de leurs rôles dans l'intégration scolaire d'un élève dysphasique en classe ordinaire au primaire », Revue des Sciences de l'Éducation, 32(3), p. 545-566.

Beckman, P. (2003). «Relations parents et professionnels dans des structures éducatives inclusives », Éducation et francophonie, 31(1). [http://www.acelf.ca/revue/31-1/index.html.]

Beveridge, S. (2005). Children, Families and Schools: Developing Partnerships for Inclusive Education, Londres et New York, Routledge Falmer.

Blue-Banning, M., J. A. Summers, H. C. Frankland, L. L. Nelson et G. Beegle (2004). «Dimensions of family and professional partnerships: Constructive guidelines for collaboration », Exceptional Children, 70(2), p. 167-184.

Bouchard, J.-M. et J.-C. Kalubi (2003). «Les difficultés de communication entre intervenants et parents d'enfants vivant avec des incapacités », Éducation et francophonie, 31(1), p. 108-129.

Centre de réadaptation de l'Estrie (CRE). (2006). Projet Apollo, document de travail, Sherbrooke (Québec), CRE.

Creswell, J. (2002). Educational Research: Planning, Conducting, and Evaluating Quantitative and Qualitative Research, Upper Saddle River (NJ), Merrill.

Denzin, N. et Y. Lincoln (2005). The Sage Handbook of Qualitative Research, Londres, Sage Publications.

Desaulniers, M., P. Beaupré, C. Lepage et E. Granger (2005). « Analyse des besoins des parents et des enseignants reliés à l'intégration scolaire des enfants ayant subi un traumatisme cranio-cérébral », Différences, 5(2), p. 40-46.

Dinnebeil, L. A., L. M. Hale et S. Rule (1996). "A qualitative analysis of parents' and service coordinators' descriptions of variables that influence collaborative relationships », Topics in Early Chilhood Special Education, 16(3), p. 322-347.

Eppler, C. et S. Weir (2009). " Family assessment in K-12 settings: Understanding family systems to provide effective, collaborative services », Psychology in the Schools, 46(6), p. 501-515.

Epstein, J. (2001). School, Family and Community Partnerships, Boulder, Westview Press.

Normand-Guérette, D. et L. Frénette (1993). «Besoin de perfectionnement des enseignants travaillant auprès d'élèves déficients intellectuels profonds multihandicapés », dans Actes du $11 I^{e}$ Congrès de l'A.I.R.H.M., Trois-Rivières (Québec), p. 355-359.

Paillé, P. et A. Mucchielli (2008). L'analyse qualitative en sciences humaines et sociales, Paris, Armand Colin, $1^{\text {re }}$ éd. 2003.

Pineault, R. et C. Daveluy (1995). La planification de la santé : concepts, méthodes, stratégies, Montréal (Québec), Éditions Nouvelles. 
Poursanidou, K., P. Garner et A. Watson (2008). « Hospital-school liaison: perspectives of health and educational professionals supporting children with renal transplants », Journal of Children Health Care, 13(4), p. 253-267.

Pratkanis, A. R., S. J. Breckler et A. G. Greenwald (dir.) (1989). Attitude, Structure and Function, Hillsdale $(\mathrm{NJ})$, Lawrence Erlbaum Associates Inc.

Saaranen, T., K. Tossavainen et H. Turunen (2005). " School staff members' and occupational health nurses' evaluation of the promotion of occupational well-being - with good planning to better practice », Journal of Interprofessional Care, 19(5), p. 465-479.

Savoie-Zajc, L. (2000). «La recherche qualitative/interprétative en éducation », dans T. Karsenty et L. Savoie-Zjac (dir.), Introduction à la recherche, Sherbrooke (Québec), Éditions CRP, p. 171-198.

Sezgin, F. (2009). "Examining the relationship between teacher organizational commitment and school health in Turkish primary schools », Educational Research and Evaluation, 15(2), p. 185201.

Sicotte, C., D. D'Amour et M. P. Moreault (2002). «Interdisciplinary collaboration within Quebec Community Health Care Centres », Social Science \& Medecine, 55(6), p. 991-1003.

St-Onge, M., V. Béguet et P. Fougeyrollas (2002). "Qualité et conditions de collaboration avec les familles perçues par le personnel de réadaptation en milieu psychiatrique et de déficience physique », Revue canadienne de santé mentale communautaire, 21(1), p. 115-135.

Turnbull, A., H. R. Turnbull, E. Erwin et L. Soodak (2006). Families, Professionals, and Exceptionality: Positive Outcomes through Partnerships and Trust, Upper Saddle River (NJ), Pearson et Merrill Prentice Hall.

Ulrich, M. E. et A. M. Bauer (2003). «Levels of awareness: A closer look at communication between parents and professionals », Teaching Exceptional Children, 35(6), p. 20-24.

Van der Maren, J.-M. (1995). Méthodes de recherche pour l'éducation, Montréal (Québec), Les Presses de l'Université de Montréal. 